\title{
Über die Isotopenzusammensetzung und über den Sitz der leichten Uredelgase in Steinmeteoriten
}

\author{
H. Hintenberger, E. Vilcsek und H. Wänke \\ Max-Planck-Institut für Chemie (Otto-Hahn-Institut), Mainz \\ (Z. Naturforschg. 20 a, 939-945 [1965]; eingegangen am 13. April 1965)
}

\begin{abstract}
Using newly developed techniques for the separate measurements of rare gases in different mineral components of stone meteorites, comprehensive investigations were carried out on meteorites containing primordial rare gases. For the bronzite chondrites $\mathrm{B} \mathrm{re} \mathrm{its} \mathrm{c} \mathrm{h} \mathrm{e} \mathrm{i} \mathrm{d} \mathrm{and} \mathrm{Pant}$ a $\mathrm{r}$, we could prove that all main mineral components contain light primordial gases, probably this is also true for the carbonaceous chondrite $\mathrm{Murray}$ and for the achondrite $\mathrm{K}$ a p o e $\mathrm{ta}$. The light primordial gases are highly concentrated in the outmost layers of the single mineral grains. The ratios for ${ }^{4} \mathrm{He} /{ }^{3} \mathrm{He}$ and ${ }^{20} \mathrm{Ne} /{ }^{22} \mathrm{Ne}$ vary for different meteorites and also for different mineral components within a single meteorite. Both ratios shows a correlation with the diffusion coefficients of the minerals involved; the lighter isotope being always more depleted in those minerals with the higher diffusion losses. According to the observed diffusion losses, the original primordial Helium content of Pantar comes out to be at least about two orders of magnitude higher than at present. For the true and original elemental and isotopic ratios we obtained values of 13.8 and 14.0 for ${ }^{20} \mathrm{Ne} /{ }^{22} \mathrm{Ne}, 2200$ for ${ }^{4} \mathrm{He} /{ }^{3} \mathrm{He}$ and 800 for ${ }^{4} \mathrm{He} /{ }^{20} \mathrm{Ne}$. These ratios have been altered considerably on account of gas losses by diffusion.
\end{abstract}

Seit dem ersten Auffinden der sogenannten Uredelgase in Meteoriten durch GerLing und LevskiI ${ }^{1}$ im Meteorit $\mathrm{Pes}$ y a n o sind eine Reihe weitere uredelgashaltige Meteorite entdeckt worden ${ }^{2-14}$. Zunächst schien es, als würden Uredelgase nur in seltenen Klassen von Meteoriten auftreten. Es zeigte sich, daß viele kohlige Chondrite sowie einige Enstatit-Chondrite größere Mengen von Uredelgasen enthielten. In unserer Diskussion wollen wir uns dabei auf solche Meteorite beschränken, die größere Mengen leichter Uredelgase, insbesondere Helium und Neon, enthalten. Die schweren Uredelgase, Krypton und Xenon, wurden in unterschiedlichen Mengen in vielen Meteoriten nachgewiesen ${ }^{8,10,15}$

Im Laufe systematischer Untersuchungen über den Edelgasgehalt von Meteoriten, wie wir sie in unserem Institut seit einigen Jahren durchführen, fanden wir auch in einer Reihe ganz gewöhnlicher

1 E. K. Gerling u. L. K. Levskir, Dokl. Akad. Nauk, SSSR 111, 750 [1956].

2 J. H. Reynolds, Phys. Rev. Letters 4, 351 [1960].

3 J. Z̈̈hringer u. W. Gentner, Z. Naturforschg. 15 a, 600 [1960].

4 H. Stauffer, Geochim. Cosmochim. Acta 24, 70 [1961].

5 H. König, K. Keil, H. Hintenberger, F. Wlotzka u. F. BegeMaNN, Z. Naturforschg. 16 a, 1124 [1961].

6 H. Hintenberger, H. König u. H. Wänke, Z. Naturforschg. 17 a, 306 [1962].

7 H. König, K. Keil u. H. Hintenberger, Z. Naturforschg. 17 a, 357 [1962].

8 J. Z̈̈̈ringer, Z. Naturforschg. 17 a, 460 [1962].

9 J. Zähringer, Geochim. Cosmochim. Acta 26, 665 [1962].
Chondrite größere Mengen von leichten Uredelgasen ${ }^{5-7,13,14}$. Stets war, wie zuerst in der Arbeit von König, Keil, Hintenberger, Wlotzka und BegeMANN ${ }^{5}$ hervorgehoben wurde, der Uredelgasgehalt mit einer sogenannten Hell-Dunkel-Struktur der Meteorite verbunden. Nur die dunklen Anteile der Meteorite enthielten große Mengen leichter Uredelgase, in den hellen Anteilen waren bisher keine leichten Uredelgase nachweisbar. Wie aus der Arbeit von Signer und Suess ${ }^{10}$ hervorgeht, gilt dies jedoch nicht für die schweren Uredelgase, obwohl auch hier die Konzentrationen in den dunklen Anteilen höher sind. Wir haben in folgenden Meteoriten leichte Uredelgase gefunden: $\mathrm{P}$ antar ${ }^{5}$, Breitscheid ${ }^{6}$, T abor ${ }^{7}$ und Pultusk ${ }^{13}$ und in allerletzter Zeit ferner in: Tysnes Island, $\mathrm{Nulles}, \mathrm{H}$ ainaut, Canges de Onis, Kilb o u rn und We ston. Manuel und Kuroda ${ }^{12}$ so-

10 P. Signer u. H. E. Suess, Rare Gases in the Sun, in the Atmosphere, and in Meteorites, in Earth Sciences and Meteoritics, Ed. J. Geiss u. E. D. Goldberg, North Holland Publ. Co., Amsterdam 1963, p. 241.

11 P. Signer, private Mitteilung.

12 O. K. Manuel u. P. K. Kuroda, J. Geophys. Res. 69, 1413 [1964].

13 H. Hintenberger, H. König, L. Schultz u. H. Wänke, Z. Naturforschg. 19 a, 327 [1963].

14 H. Hintenberger, L. Schultz u. H. Wänke, Z. Naturforschg. 20 a [1965], in Vorbereitung.

15 D. Krummenacher, C. M. Merrihue, R. O. Pepin u. J. H. Reynolds, Geochim. Cosmochim. Acta 26, 231 [1962]. 
wie Signer und Suess ${ }^{11}$ entdeckten, daß auch der Meteorit F a y t t e ville Uredelgase enthielt. Dieser hat den weitaus höchsten Gehalt an Urhelium. Alle elf Meteorite gehören in die Klasse der BronzitChondrite. Hierbei haben wir nur solche Meteorite aufgezählt, die beobachtete Fälle sind.

Seit dem Auffinden der Uredelgase gab es auch zahlreiche Arbeiten zur Deutung dieses Phänomens (vgl. 1, 9,16-18). Wir wollen hier weniger auf die verschiedenen in diesem Zusammenhang aufgestellten Theorien eingehen, als vielmehr die dabei verwendeten Methoden kritisch beleuchten.

Die Diffusionsversuche von Z̈̈HRINGER ${ }^{9}$ sowie unveröffentlichte von uns selbst durchgeführte zeigten, $\mathrm{da} ß$ ein großer Teil der leichten Uredelgase aus den Meteoriten schon bei geringerer Temperatur abgegeben wird als die radiogenen und spallogenen (durch die Einwirkung der Höhenstrahlung in Spallationsreaktionen gebildeten) Edelgase.

Untersuchungen über den Sitz der Uredelgase wie sie vor allem von ZäHRINGER ${ }^{9}$ sowie von KöNIG und W $_{\text {LOTZKA }}{ }^{18}$ angestellt wurden, lieferten kein eindeutiges Bild. Alle Hauptmineralbestandteile der Meteorite erwiesen sich als praktisch frei von Uredelgasen. Hierbei ist zu bemerken, daß durch die für solche Untersuchungen verwendeten klassischen Methoden der Mineraltrennung von vornherein eine große Einschränkung gegeben war, die manche Irreführung verursachte. Rein mechanische Trennungen durch Aussortieren beschränken sich naturgemäß immer nur auf einige extrem große Kristalle. Bei den weiteren allgemein üblichen Methoden der Mineraltrennung hat man es bei den getrennten Kristallen meist mit solchen von einheitlicher Korngröße zu tun, vor allem aber bleiben häufig alle feinkörnigen Anteile ungetrennt. In den Versuchen von KöNIG und Wlotzka hatten diese feinkörnigen, ungetrennten Anteile sowohl die magnetischen als auch die unmagnetischen stets hohe Mengen von Uredelgasen enthalten. Wie wir später noch zeigen werden, sitzen die leichten Uredelgase stets nur in den äußeren Schichten der einzelnen Mineralkörner und sind daher auf Grund des Verhältnisses von

16 E. R. DuFresne u. E. Anders, Geochim. Cosmochim. Acta 26, 251 [1962].

17 K. Frederiksson u. K. Keil, Geochim. Cosmochim. Acta 27, 717 [1963].

18 H. König u. F. WLotzka, Z. Naturforschg. 17 a, 472 [1962].
Oberfläche zu Volumen im feinkörnigen Material angereichert *

\section{Versuche mit Lösungsextraktionen}

Wir haben deshalb ein Verfahren entwickelt, bei dem stets die ganze Untersuchungsprobe unabhängig von der Korngröße zur Edelgasmessung ausgenützt wurde.

Durch aufeinanderfolgende Behandlung der pulverisierten Meteoritprobe mit verschiedenen Lösungsflüssigkeiten konnten die entsprechenden Phasen in Lösung gebracht, und die in ihnen enthaltenen Edelgase getrennt gemessen werden. Für die einzelnen Schritte wurden eine $\mathrm{FeCl}_{3}$ - bzw. $\mathrm{CuCl}_{2}$ Lösung zum Lösen des metallischen Nickeleisens, Bromwasser zum Aufschluß des Troilits und Salzsäure zur Lösung des Olivins verwendet. Nach dem Einwirken der einzelnen Lösungsflüssigkeiten wurde die jeweils ungelöst gebliebene Probe von der Lösung abgetrennt, und die in Lösung gegangenen Elemente chemisch bestimmt. Aus dem in allen diesen Flüssigkeiten unlöslich gebliebenen Rückstand wurden die Edelgase durch Erhitzen ausgetrieben. Die Details dieses Verfahrens werden ausführlich in einer Arbeit von VILcsek und WäNke ${ }^{19}$ beschrieben, so daß es sich erübrigt, hier näher darauf einzugehen.

Die Messung der extrahierten Edelgase erfolgt in der schon mehrmals von uns beschriebenen Weise ${ }^{13,20}$. Die Ergebnisse dieser Analysen an einigen Meteoriten sind in Tab. 1 festgehalten. Mit Ausnahme der Messungen am kohligen Chondriten M u r r a y und $\mathrm{P}$ a n t a 5 liegen diese Versuche schon längere Zeit zurück. Wir haben unser Verfahren inzwischen weiter vervollkommnet; die Ergebnisse sind jedoch im großen und ganzen dieselben wie wir sie in den späteren Versuchen (P a n t a r 5) erhielten. In weiteren Versuchen haben wir vor der Anwendung der Lösungstechnik eine Vortrennung entweder nach der Korngröße oder eine Zerlegung in eine magnetische und eine unmagnetische Fraktion vorgenommen.

* A n m. b. d. K o r r. : Für die Silikatkristalle des uredelgashaltigen Achondriten $\mathrm{K}$ h or $\mathrm{T}$ e mik i fanden EBERhaRdT, Geiss und Grögler, wie eben bekannt wurde, ähnliche Ergebnisse (Vorabdruck Februar und April 1965).

19 E. Vilcsek u. H. Wänke, Z. Naturforschg. 20 a [1965], in Vorbereitung.

20 K. H. Ebert u. H. Wänke, Z. Naturforschg. 12 a, 766 [1957]. 


\begin{tabular}{|c|c|c|c|c|c|c|c|c|c|c|c|c|c|}
\hline $\begin{array}{c}\text { Meteorit } \\
\text { Extraktions- } \\
\text { verfahren }\end{array}$ & $\begin{array}{l}\text { Gelöstes } \\
\text { Material } \\
\text { in } \mathrm{mg} / \mathrm{g}\end{array}$ & $\begin{array}{c}\Sigma \mathrm{He} \\
10^{-8} \\
\mathrm{~cm}^{3} / \mathrm{g}\end{array}$ & $\begin{array}{c}\Sigma \mathrm{Ne} \\
10^{-8} \\
\mathrm{~cm}^{3} / \mathrm{g}\end{array}$ & $\begin{array}{c}\Sigma \mathrm{He} \\
10^{-8} \\
\mathrm{~cm}^{3} / \mathrm{g}^{*}\end{array}$ & $\frac{\Sigma \mathrm{He}}{\Sigma \mathrm{Ne}}$ & $\frac{{ }^{4} \mathrm{He}}{{ }^{3} \mathrm{He}}$ & $\frac{20 \mathrm{Ne}}{22 \mathrm{Ne}}$ & $\frac{22 \mathrm{Ne}}{21 \mathrm{Ne}}$ & $\begin{array}{c}{ }^{3} \mathrm{He} \\
10^{-8} \\
\mathrm{~cm}^{3} / \mathrm{g}^{*}\end{array}$ & $\begin{array}{c}\text { Spall. } \\
{ }^{3} \mathrm{He} \\
10^{-8} \\
\mathrm{~cm}^{3} / \mathrm{g}^{*}\end{array}$ & $\begin{array}{l}\frac{{ }^{4} \mathrm{He}}{} \\
{ }^{3} \mathrm{He} \\
\text { corr. }\end{array}$ & $\begin{array}{c}\text { Spall. } \\
21 \mathrm{Ne} \\
10^{-8} \\
\mathrm{~cm}^{3} / \mathrm{g}^{*}\end{array}$ & $\begin{array}{l}\frac{20 \mathrm{Ne}}{22 \mathrm{Ne}} \\
\text { corr. }\end{array}$ \\
\hline \begin{tabular}{|lr} 
Breits cheid \\
$\mathrm{H}_{2} \mathrm{O}$ & $3 \mathrm{~h}$ \\
$\mathrm{FeCl}_{3}$ & $7 \mathrm{~h}$ \\
$\mathrm{Br}_{2}$ & $1 \mathrm{~h}$ \\
$\mathrm{HCl}$ & $13 \mathrm{~h}$ \\
Rückst. & $1 \mathrm{~h}$ \\
$\Sigma$ & \\
\end{tabular} & \begin{tabular}{ll} 
& \multicolumn{2}{c}{$\mathrm{M}^{-}+200$} \\
$56 \mathrm{FeS}+66 \mathrm{O}$ \\
$194 \mathrm{O}$ \\
$498 \mathrm{P}$ \\
1011
\end{tabular} & $\begin{array}{r}490 \\
5040 \\
1790 \\
1120 \\
1670 \\
10110\end{array}$ & \begin{tabular}{|r|}
9,0 \\
38,7 \\
15,8 \\
17,2 \\
50,4 \\
131,1
\end{tabular} & $\begin{array}{r}- \\
25600 \\
14700 \\
5780 \\
3340\end{array}$ & $\begin{array}{r}54 \\
129 \\
116 \\
65 \\
32\end{array}$ & $\begin{array}{r}1775 \\
492 \\
750 \\
70 \\
202\end{array}$ & \begin{tabular}{|c|}
14,2 \\
12,7 \\
8,0 \\
2,04 \\
11,15
\end{tabular} & $\begin{array}{l}- \\
7,76 \\
2,56 \\
1,18 \\
1,83\end{array}$ & $\begin{array}{l}5 \overline{2} \\
19,6 \\
82 \\
16,5\end{array}$ & $\begin{array}{l}\overline{50} \\
\overline{-} \\
67 \\
14,5\end{array}$ & $\begin{array}{l}- \\
- \\
- \\
-\end{array}$ & $\begin{array}{l}\overline{1}, 33 \\
4,99 \\
19,4 \\
7,14\end{array}$ & $\begin{array}{r}14,2 \\
14,1 \\
12,7 \\
-\overline{13}, 0\end{array}$ \\
\hline $\begin{array}{ll}\text { Pantar } 2 \\
\mathrm{FeCl}_{3} & 6 \mathrm{~h} \\
\mathrm{Br}_{2} & 2 \mathrm{~h} \\
\mathrm{HCl} & 3 \mathrm{~h} \\
\text { Rückst. } & 1 \mathrm{~h} \\
\Sigma & \end{array}$ & $\begin{array}{l}\sim 200 \mathrm{M}+17 \mathrm{O} \\
\sim 60 \mathrm{FeS}+103 \mathrm{O} \\
105 \mathrm{O} \\
\quad 440 \mathrm{P} \\
\quad 925\end{array}$ & $\begin{array}{r}36200 \\
12900 \\
1460 \\
11900 \\
62460\end{array}$ & $\begin{array}{r}78,5 \\
40,0 \\
5,7 \\
45,2 \\
169,4\end{array}$ & $\begin{array}{r}165000 \\
79000 \\
13900 \\
27000\end{array}$ & $\begin{array}{l}462 \\
324 \\
256 \\
265\end{array}$ & $\begin{array}{l}2800 \\
3140 \\
1340 \\
1950\end{array}$ & $\begin{array}{l}12,83 \\
11,3 \\
8,65 \\
10,1\end{array}$ & $\begin{array}{l}27 \\
12,9 \\
3,23 \\
8,20\end{array}$ & $\begin{array}{l}59 \\
25 \\
10,4 \\
14\end{array}$ & $\begin{array}{l}2,8 \\
1,5 \\
5,9 \\
3,6\end{array}$ & $\begin{array}{l}2900 \\
3400 \\
3100 \\
2600\end{array}$ & $\begin{array}{l}-\overline{8} \\
0,87 \\
1,55 \\
0,83\end{array}$ & $\begin{array}{l}12,9 \\
11,8 \\
12,3 \\
11,0\end{array}$ \\
\hline 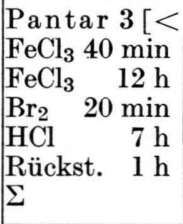 & $\begin{array}{l}60 \mu](=71 \%) \\
\sim 100 \mathrm{M}+20 \mathrm{O} \\
\sim 70 \mathrm{M}+30 \mathrm{O} \\
\sim 60 \mathrm{FeS}+63 \mathrm{O} \\
\quad 193 \mathrm{O} \\
\quad 474 \mathrm{P} \\
1010\end{array}$ & $\begin{array}{r}15300 \\
27900 \\
2870 \\
4710 \\
12700 \\
63480\end{array}$ & \begin{tabular}{r|}
29,9 \\
70,4 \\
8,2 \\
16,2 \\
49,3 \\
174,0
\end{tabular} & $\begin{array}{r}127000 \\
279000 \\
23300 \\
24400 \\
26800\end{array}$ & $\begin{array}{l}511 \\
393 \\
319 \\
285 \\
257\end{array}$ & $\begin{array}{l}2110 \\
3600 \\
1690 \\
3240 \\
2200\end{array}$ & $\begin{array}{c}11,7 \\
12,0 \\
10,2 \\
8,5 \\
9,96\end{array}$ & $\begin{array}{c}25,7 \\
21,4 \\
10,9 \\
4,31 \\
8,13\end{array}$ & $\begin{array}{r}60,3 \\
77,0 \\
13,8 \\
7,5 \\
12,2\end{array}$ & $\begin{array}{l}2,8 \\
2,8 \\
1,5 \\
5,9 \\
3,6\end{array}$ & $\begin{array}{c}2200 \\
3800 \\
1900 \\
- \\
3100\end{array}$ & $\begin{array}{l}-\overline{6} 6 \\
0,41 \\
1,8 \\
0,86\end{array}$ & $\begin{array}{l}11,8 \\
12,2 \\
10,8 \\
10,4 \\
10,9\end{array}$ \\
\hline \begin{tabular}{|lr} 
Pantar $3[>$ \\
$\mathrm{FeCl}_{3}$ & $40 \mathrm{~min}$ \\
$\mathrm{FeCl}_{3}$ & $10 \mathrm{~h}$ \\
$\mathrm{Br}_{2}$ & $20 \mathrm{~min}$ \\
$\mathrm{HCl}$ & $6 \mathrm{~h}$ \\
Rückst. & $1 \mathrm{~h}$ \\
$\Sigma$ &
\end{tabular} & $\begin{array}{l}60 \mu](=29 \%) \\
\sim 110 \mathrm{O} \\
290 \mathrm{M}+21 \mathrm{O} \\
60 \mathrm{FeS}+64 \mathrm{O} \\
71 \mathrm{O} \\
330 \mathrm{P} \\
946\end{array}$ & $\begin{array}{r}14100 \\
8760 \\
6130 \\
3880 \\
11800 \\
44670\end{array}$ & \begin{tabular}{|r|}
18,9 \\
26,9 \\
13,6 \\
15,9 \\
44,5 \\
119,8
\end{tabular} & $\begin{array}{r}128000 \\
28200 \\
49500 \\
54500 \\
35800\end{array}$ & $\begin{array}{l}745 \\
326 \\
450 \\
244 \\
265\end{array}$ & $\begin{array}{l}2100 \\
3400 \\
3800 \\
2000 \\
1400\end{array}$ & $\begin{array}{l}13,70 \\
12,50 \\
12,55 \\
10,4 \\
11,1\end{array}$ & $\begin{array}{c}23,8 \\
26,9 \\
14,5 \\
7,40 \\
10,8\end{array}$ & $\begin{array}{r}61,0 \\
8,3 \\
14,6 \\
27 \\
26\end{array}$ & $\begin{array}{l}2,8 \\
2,8 \\
1,5 \\
5,9 \\
3,6\end{array}$ & $\begin{array}{l}2200 \\
5100 \\
3800 \\
2600 \\
1600\end{array}$ & $\begin{array}{l}- \\
\overline{-} \\
0,27 \\
2,0 \\
0,68\end{array}$ & $\begin{array}{l}13,8 \\
12,5 \\
12,8 \\
11,6 \\
11,7\end{array}$ \\
\hline $\begin{array}{ll}\text { Pantar } & 4 \text { (ma } \\
\mathrm{FeCl}_{3} & 1 \mathrm{~h} \\
\mathrm{FeCl}_{3} & 3 \mathrm{~h} \\
\mathrm{Br}_{2} & \\
\text { Rückst. } & \\
\end{array}$ & $\begin{array}{l}\text { gnetisch) } \\
\sim 220 \mathrm{M}+5,4 \mathrm{O} \\
\sim 370 \mathrm{M}+15 \mathrm{O} \\
22 \mathrm{FeS}+44 \mathrm{O} \\
296 \mathrm{P}+\mathrm{O}\end{array}$ & $\begin{array}{r}36200 \\
14400 \\
2650 \\
-\end{array}$ & $\begin{array}{c}54,1 \\
44,9 \\
9,3 \\
-\end{array}$ & $\begin{array}{r}161000 \\
37400 \\
40200 \\
-\end{array}$ & $\begin{array}{r}669 \\
322 \\
285 \\
-\end{array}$ & $\begin{array}{c}2430 \\
2280 \\
3210 \\
-\end{array}$ & $\begin{array}{c}13,37 \\
12,58 \\
11,58 \\
- \\
\end{array}$ & $\begin{array}{l}26,1 \\
25,6 \\
20,32 \\
- \\
\end{array}$ & $\begin{array}{l}66,3 \\
16,4 \\
12,5 \\
- \\
\end{array}$ & $\begin{array}{l}2,8 \\
2,8 \\
1,5 \\
- \\
\end{array}$ & $\begin{array}{c}2500 \\
2800 \\
3700 \\
- \\
\end{array}$ & $\begin{array}{l}- \\
\overline{0}, 18 \\
-\end{array}$ & $\begin{array}{l}13,4 \\
12,7 \\
11,8 \\
-\end{array}$ \\
\hline $\begin{array}{ll}\mathrm{Pantar}^{2} & 5 \\
\mathrm{CuCl}_{2} & 25 \mathrm{~min} \\
\mathrm{Br}_{2} & 10 \mathrm{~min} \\
\mathrm{HCl} & 4 \mathrm{~h} \\
\text { Rückst. } & 1 \mathrm{~h} \\
\Sigma & \end{array}$ & $\begin{array}{l}171 \mathrm{M}+7 \mathrm{O} \\
52 \mathrm{FeS}+36 \mathrm{O} \\
285 \mathrm{O} \\
456 \mathrm{P} \\
1007\end{array}$ & $\begin{array}{r}14500 \\
16900 \\
10900 \\
9520 \\
51820\end{array}$ & $\begin{array}{r}23,2 \\
36,0 \\
34,1 \\
26,8 \\
120,1\end{array}$ & $\begin{array}{r}81400 \\
192000 \\
38200 \\
20900\end{array}$ & $\begin{array}{l}623 \\
470 \\
327 \\
353\end{array}$ & $\begin{array}{c}- \\
3050 \\
2360 \\
-\end{array}$ & $\begin{array}{c}- \\
\overline{-} \\
10,19 \\
-\end{array}$ & $\begin{array}{l}- \\
- \\
6,26 \\
-\end{array}$ & $\begin{array}{l}- \\
63,0 \\
16,2 \\
-\end{array}$ & $\begin{array}{l}- \\
1,5 \\
5,9 \\
-\end{array}$ & $\begin{array}{c}- \\
3100 \\
3700 \\
-\end{array}$ & $\begin{array}{c}- \\
\overline{-} \\
1,35 \\
-\end{array}$ & $\begin{array}{l}- \\
\overline{11}, 7 \\
-\end{array}$ \\
\hline $\begin{array}{ll}\text { Kapoeta } \\
\mathrm{H}_{2} \mathrm{O} & 1 \mathrm{~h} \\
\mathrm{FeCl}_{3} & 6 \mathrm{~h} \\
\mathrm{Br}_{2} & 1 \mathrm{~h} \\
\mathrm{HCl} & 1 \mathrm{~h} \\
\text { Rückst. } & 1 \mathrm{~h} \\
\Sigma & \end{array}$ & 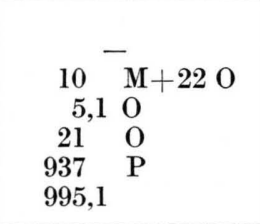 & $\begin{array}{r}240 \\
28100 \\
- \\
2750 \\
93200 \\
124290\end{array}$ & $\begin{array}{r}-\overline{9} \\
-\overline{-} \\
65 \\
580 \\
1640\end{array}$ & $\begin{array}{c}-\overline{-} \\
877000 \\
- \\
131000 \\
99500\end{array}$ & $\begin{array}{r}36 \\
28 \\
-\overline{42} \\
160\end{array}$ & $\begin{array}{l}\overline{2920} \\
- \\
2600 \\
2300\end{array}$ & $\begin{array}{l}- \\
12,3 \\
- \\
12,6 \\
11,86\end{array}$ & $\begin{array}{l}\overline{-} \\
26,2 \\
\overline{1} \\
16,2 \\
12,85\end{array}$ & $\begin{array}{r}300 \\
\overline{-} \\
40,3 \\
43,3\end{array}$ & $\begin{array}{l}- \\
- \\
- \\
-\end{array}$ & $\begin{array}{l}- \\
- \\
- \\
-\end{array}$ & $\begin{array}{l}- \\
\overline{-} \\
6,4 \\
2,3\end{array}$ & $\begin{array}{l}\overline{12,4} \\
\overline{12}, 9 \\
12,4\end{array}$ \\
\hline $\begin{array}{l}\text { Murray } \\
\mathrm{CuCl}_{2} \quad 30 \text { min } \\
\mathrm{Br}_{2} \quad 15 \mathrm{~min} \\
\mathrm{HCl} \quad 2,5 \mathrm{~h} \\
\text { Rückst. } \quad 1 \mathrm{~h} \\
\Sigma\end{array}$ & $\begin{array}{r}30 \mathrm{a} \\
68 \mathrm{~b} \\
\sim 320 \mathrm{c} \\
482 \mathrm{~d} \\
900\end{array}$ & $\begin{array}{r}60 \\
1300 \\
3510 \\
5320 \\
10190\end{array}$ & $\begin{array}{r}1,2 \\
3,1 \\
25,8 \\
18,3 \\
48,4\end{array}$ & $\begin{array}{r}2000 \\
19100 \\
10600 \\
11300\end{array}$ & $\begin{array}{l}49,5 \\
428 \\
136 \\
291\end{array}$ & $\begin{array}{l}1470 \\
2210 \\
2550 \\
1780\end{array}$ & $\begin{array}{c}- \\
12,0 \\
9,67 \\
7,47\end{array}$ & $\begin{array}{l}-\overline{8,9} \\
5,41 \\
4,85\end{array}$ & $\begin{array}{l}1,4 \\
8,7 \\
4,2 \\
6,4\end{array}$ & $\begin{array}{l}- \\
- \\
-\end{array}$ & $\begin{array}{l}- \\
- \\
-\end{array}$ & $\begin{array}{l}-\overline{2} 9 \\
1,15 \\
0,81\end{array}$ & $\begin{array}{r}\overline{13}, 0 \\
11,3 \\
9,0\end{array}$ \\
\hline
\end{tabular}

Tab. 1. Edelgasdaten der Lösungsversuche. $\mathrm{cm}^{3} / \mathrm{g} *$ bedeutet Edelgasmenge pro Gramm gelöstes Material. $\mathrm{M}=\mathrm{Metall}$, $\mathrm{O}=\mathrm{Oli}-$ vin, $\mathrm{P}=$ Pyroxen (+ Feldspäte), $\mathrm{FeS}=$ Troilit. Bei dem kohlenstoffhaltigen Meteoriten $\mathrm{M} \mathrm{u} \mathrm{r} \mathrm{r}$ a y ist die Zuordnung der in Lösung gegangenen Elemente zu bestimmten Mineralen nicht eindeutig. Hier steht a für Metall+Silikat, b für Schwefel (gebunden oder frei) +Eisenoxyd + Silikat, c und d für Silikate. 


\section{Deutung der Ergebnisse der Lösungs- extraktionen}

Wie aus Tab. 1 ersichtlich, lieferten die einzelnen Phasen starke Unterschiede in den Isotopenverhältnissen. Die höchsten ${ }^{20} \mathrm{Ne} /{ }^{22} \mathrm{Ne}$ - sowie die niedrigsten ${ }^{4} \mathrm{He} /{ }^{3} \mathrm{He}-$ Verhältnisse (stets für Spallationsanteile korrigiert) ergaben sich immer in den $\mathrm{FeCl}_{3}$ bzw. $\mathrm{CuCl}_{2}$-Fraktionen, welche der Nickeleisenphase entsprechen. Bei den Versuchen Pantar 3 haben wir die $\mathrm{FeCl}_{3}$-Lösung zunächst nur kurze Zeit einwirken lassen, um möglichst reine Eisennickelphase zu lösen. (Der Hauptteil der Eisennickelphase ist stets bereits nach etwa $40 \mathrm{~min}$ gelöst, jedoch bleiben einige größere Körner übrig, die sich nur langsam lösen.) Bei der grobkörnigen Fraktion $\mathrm{P}$ a n $\operatorname{tar} 3[>60 \mu]$ ist dies auch sehr gut gelungen. Bei dieser Fraktion zeigt sich auch mit großer Deutlichkeit, daß die Uredelgase stets nur in den äußersten Schichten der einzelnen Mineralkörner sitzen. $\mathrm{FeCl}_{3}$ [40 min] ergab beinahe fünfmal soviel Helium wie $\mathrm{FeCl}_{3}[10 \mathrm{~h}]$; wobei im letzteren Fall wohl die Hauptmenge der Edelgase den mitgelösten äußeren Schichten der Olivinkörner zuzuordnen ist und nicht dem Metall. (Die Versuche $\mathrm{FeCl}_{3}$ [40 min] und $\mathrm{FeCl}_{3}[10 \mathrm{~h}]$ wurden nacheinander mit derselben Probe ausgeführt.) Die unterschiedlichen korrigierten ${ }^{20} \mathrm{Ne} /{ }^{22} \mathrm{Ne}$ - und ${ }^{4} \mathrm{He} /{ }^{3} \mathrm{He}-$ Verhältnisse demonstrieren dies deutlich. Die Fraktion Pantar 3 $[<60 \mu]$ ergab hingegen die Hauptedelgasmenge bei $\mathrm{FeCl}_{3}[12 \mathrm{~h}]$, obwohl mehr als die Hälfte des Metalls bereits gelöst war. Der überwiegende Teil der in dieser Phase freigesetzten Edelgase entstammt sicher wieder den äußeren Schichten der in dieser Fraktion P a n t a r $3[<60 \mu]$ angereicherten Olivinkörner.

Wie wir in anderen Versuchen gefunden haben, sind für den Troilit die höchsten Diffusionsverluste zu erwarten ${ }^{21,22}$. In der Bromphase lösten sich neben dem Troilit stets etwa gleich große Mengen Olivin. Rückstand sowie HCl-Phase enthielten nicht immer reinen Pyroxen bzw. Olivin (besonders z. B. bei Pantar $3[>60 \mu])$. Zusammengebackene Kristalle können im Inneren teilweise noch Metall bzw. Troilit enthalten. Sowohl in der HCl-Phase als auch im Rückstand fanden sich häufig noch geringe Spuren von Nickel und von FeS.

21 H. Hintenberger, E. Vilcsek u. H. Wünke, Z. Naturforschg. 19 a, 219 [1963].
Das auf Spallationsneon korrigierte ${ }^{20} \mathrm{Ne} /{ }^{22} \mathrm{Ne}-$ Verhältnis im Nickeleisen ergab sich zu 13,8, im Olivin zu 12,2 (P a n tar $3\left[<60 \mu, \mathrm{FeCl}_{3} 12 \mathrm{~h}\right]$ ) und zu ca. 10,7 für den Pyroxen. Die höheren Werte im Rückstand von P a n t a r $3[>60 \mu]$ sind teilweise auf die vorhin erwähnten Einschlüsse zurückzuführen. In den HCl-Phasen entstammt der Hauptteil der Uredelgase vermutlich nicht dem Olivin, sondern den teilweise in Lösung gegangenen Feldspäten (wie oben ausgeführt, gehen die äußeren Schichten des Olivins bereits in der $\mathrm{FeCl}_{3}$ - bzw. Bromphase in Lösung) .

Wie wir in einer anderen Arbeit zeigen, treten Diffusionsverluste der Edelgase in den einzelnen Meteoritbestandteilen in der zunehmenden Reihenfolge - Eisen - Olivin - Pyroxen - Troilit - auf. Diese Reihenfolge zeigt nach dem oben gesagten auch das ${ }^{20} \mathrm{Ne} /{ }^{22} \mathrm{Ne}$ - und das ${ }^{4} \mathrm{He} /{ }^{3} \mathrm{He}-$ Verhältnis. Letzteres ist im Nickeleisen (P a n t a r $3[>60 \mu$, $\mathrm{FeCl}_{3} 40 \mathrm{~min}$ ]) mit einem Wert von 2200 am niedrigsten.

Vergleicht man alle Versuche an $\mathrm{P}$ a n ta $\mathrm{r} 3$, so kommt man zu dem Schluß, daß in diesem Meteoriten etwa $50 \%$ des Urheliums im Olivin enthalten ist, ca. $30 \%$ sitzt im Nickeleisen und weniger als $20 \%$ im Pyroxen. Im Nickeleisen (Häufigkeit ca. 18\%) und im Olivin (Häufigkeit ca. 25\%) ist somit die Konzentration von Urhelium etwa gleich groß, während die Konzentration im Pyroxen um etwa einen Faktor 5 geringer ist.

Wir sind der Ansicht, daß der Großteil der von uns erstmalig aufgefundenen starken Unterschiede der Isotopenhäufigkeiten des Heliums und Neons in den einzelnen Mineralien hauptsächlich durch Diffusionsverluste bedingt ist. Für eine Verschiebung des ${ }^{20} \mathrm{Ne} /{ }^{22} \mathrm{Ne}-V e r h a ̈ l t n i s s e s$ von etwa $10 \%$ bzw. 20\% (von 13,8 auf 12,2 im Olivin bzw. von 13,8 auf ca. 10,7 im Pyroxen) ist unter der Voraussetzung einer $\mathrm{m}^{1 / 2}$-Abhängigkeit der Diffusionskonstanten eine mindestens 10- bzw. 100-fache Anfangskonzentration notwendig (vgl. Z̈̈HRINGER ${ }^{8}$ ). Setzt man die Anfangskonzentration von Neon im Olivin gleich 1 , so müßte sie demnach im Pyroxen gleich 2 und im Nickeleisen gleich 0,1 gewesen sein. Wenn, wie aus unseren Versuchen hervorgeht, die Uredelgase nur an der Oberfläche sitzen, so ist die Anfangskonzentration - gleiche Einbauausbeute vorausgesetzt - proportional zur vorhandenen

22 H. Hintenberger, L. Schultz u. H. Wänke, Z. Naturforschg. 20 a [1965], in Vorbereitung. 
Oberfläche und daher umgekehrt proportional zur Korngröße. Die abgeschätzten Anfangskonzentrationen dürften mit der Korngrößenverteilung vereinbar sein. Pantar dunkel muß somit ursprünglich eine um 1 bis 2 Größenordnungen höhere Konzentration an Uredelgasen gehabt haben.

Hohe Diffusionsverluste der Uredelgase können auch durch folgende Überlegung bewiesen werden. Der Pyroxen hat im Breits cheid ca. $76 \%$ des radiogenen und spallogenen Heliums verloren ${ }^{21}$. Bei Pantar beträgt der Verlust des Heliums im Pyroxen ca. $33 \%{ }^{22}$. Sowohl das spallogene als auch das radiogene Helium ist in den Pyroxenkörnern sicher annähernd homogen verteilt. Bei den nur in den äußersten Oberflächenschichten sitzenden Uredelgasen sind somit weit höhere Verluste zu erwarten.

Im Gegensatz zu dem über $20 \%$ liegenden Unterschied im ${ }^{20} \mathrm{Ne} /{ }^{22} \mathrm{Ne}-$ Verhältnis erscheinen die Unterschiede der $\Sigma \mathrm{He} / \Sigma \mathrm{Ne}$-Verhältnisse in den Fraktionen von $\mathrm{P}$ a n t a $\mathrm{r}$, die nur etwa einen Faktor 3 ausmachen, relativ zu gering. (Bereits mit einer reinen $m^{1 / 2}$-Abhängigkeit müßte die Verschiebung des $\Sigma \mathrm{He} / \Sigma \mathrm{Ne}$-Verhältnisses höher sein, wegen der unterschiedlichen Atomradien wäre eine noch wesentlich höhere Verschiebung zu erwarten.) Hier ist jedoch zu bedenken, daß wir stets mit einer Mischung von Mineralien mit sehr unterschiedlichen Korngrößen zu tun haben. Die kleinen Körner sind vermutlich beinahe frei von Helium, und in ihnen ist sicher die Verschiebung der Isotopenhäufigkeit des Neons am größten. Tatsächlich lieferte $\mathrm{P}$ a n t a r 3 $[<60 \mu] \mathrm{HCl}$ und Rückstand die niedrigsten ${ }^{20} \mathrm{Ne} /{ }^{22}$-Ne-Verhältnisse. Allerdings sollte der oben erwähnte Mechanismus erst bei wesentlich kleineren Korngrößen als $60 \mu$ wirksam werden. Es ist jedenfalls nicht notwendig anzunehmen, daß beim Neon eine zweite Komponente der Uredelgase einen entscheidenden Anteil hat, wie Signer und Suess ${ }^{10}$ vorgeschlagen haben. Jedoch hat eine solche zweite Komponente bei den schweren Edelgasen aller Wahrscheinlichkeit nach einen entscheidenden Anteil.

In allen Meteoriten, die in dieser Arbeit untersucht worden sind, einschließlich $\mathrm{K}$ a p o e t a und M u r r a y fanden sich in allen Hauptmineralen Uredelgase. Im Falle von $\mathrm{K}$ a p o e $\mathrm{ta}$ und $\mathrm{Mur} \mathrm{ray}$, die beide nur sehr wenig Nickeleisen enthalten, lieferte die $\mathrm{FeCl}_{3}$ - bzw. $\mathrm{CuCl}_{2}$-Phase nur relativ geringe Mengen Uredelgase. Es scheint besonders be- merkenswert, daß auch beim kohligen Chondriten Murray in den einzelnen Fraktionen sehr große Unterschiede des ${ }^{20} \mathrm{Ne} /{ }^{22} \mathrm{Ne}$-Verhältnisses auftraten. In diesem Meteoriten fand sich in der Bromphase der Wert von 13,0 für das korrigierte ${ }^{20} \mathrm{Ne} /{ }^{22} \mathrm{Ne}$ Verhältnis. Dieser Wert ist durchaus vergleichbar mit den Werten für dieses Verhältnis in den gewöhnlichen Chondriten. Man kann somit schließen, daß der Einbaumechanismus der Uredelgase in den kohligen Chondriten ähnlich dem in den gewöhnlichen Chondriten ist. Die niedrigen Werte des ${ }^{20} \mathrm{Ne} /{ }^{22} \mathrm{Ne}-$ Verhältnisses, die man bisher in Gesamtedelgasanalysen der kohligen Chondrite gemessen hat, können durch Diffusionsverluste erhalten werden.

\section{Weitere gezielte Versuche über den Sitz der leichten Uredelgase}

Von der Ausgangsprobe Pantar $3[<60 \mu]$ haben wir mit Hilfe von Schwereflüssigkeiten den Troilit abgetrennt. Diese Troilitfraktion wurde durch Sedimentation in eine Fraktion $5-10 \mu$ und eine Fraktion von ca. $10-60 \mu$ aufgetrennt. (Hierbei ist zu bemerken, daß das sehr feinkörnige Material bei der Trennung verlorenging.) Durch Anwendung der Lösungstechnik war es möglich, reine Troilitphase zu bekommen. Da nur die Edelgase im Troilit interessierten, wurde nur die Bromphase gemessen. In Tab. 2 sind die Edelgasgehalte dieser Fraktionen zusammen mit einer Messung von Troilit $[\geqq 30 \mu]$ von KöNIG und WLOTZKA ${ }^{18}$ aufgeführt.

\begin{tabular}{|c|c|c|c|c|}
\hline & $\begin{array}{c}\text { Troilit- } \\
\text { Korngröße }\end{array}$ & $\begin{array}{c}\sum \mathrm{He} \\
10^{-8} \mathrm{~cm}^{3} / \mathrm{g}\end{array}$ & $\begin{array}{c}\Sigma \mathrm{Ne} \\
10^{-8} \mathrm{~cm}^{3} / \mathrm{g}\end{array}$ & $\frac{\Sigma \mathrm{He}}{\Sigma \mathrm{Ne}}$ \\
\hline $\mathrm{a}$ & $5-10 \mu$ & 85.200 & 352 & 242 \\
\hline $\mathrm{b}$ & $10-60 \mu$ & 19.800 & 43,4 & 456 \\
\hline $\mathrm{c}$ & $\geqq 30 \mu$ & 3.910 & - & - \\
\hline
\end{tabular}

Tab. 2. Edelgasmessungen an abgetrenntem Troilit aus $\mathrm{P}$ a $\mathrm{n}$ t a r. a, b P a n t a r 3, c Messung von König und W $\mathrm{W}_{\text {LOtZKa }}{ }^{18}$.

Im feinkörnigen Troilit $[5-10 \mu]$ sind die Konzentrationen der leichten Uredelgase gegenüber den grobkörnigen Fraktionen stark erhöht. Ähnliche Ergebnisse wurden in einer kürzlich erschienenen Arbeit von Eberhardt, Geiss und Grögler ${ }^{23}$ gefunden.

23 P. Eberhardt, J. Geiss u. N. Grögler, Vorabdruck 1965. 
Nachdem wir schon bei den Versuchen mittels Lösungsextraktion deutliche Hinweise dafür erhalten hatten, daß die Hauptmengen der Uredelgase in den Oberflächenschichten der einzelnen Körner sitzen, haben wir mit dem unlöslichen Rückstand von $\mathrm{P}$ antar $3[<60 \mu]$ einen weiteren Versuch unternommen. Zunächst haben wir diesen Rückstand eine Stunde lang mit Königswasser bei ca. $50{ }^{\circ} \mathrm{C}$ behandelt. Hierbei ging nur ganz wenig Material in Lösung. Die im Rückstand bestimmte Edelgaskonzentration war innerhalb unserer Fehlergrenzen gleich geblieben. Wir konnten daher einigermaßen sicher sein, daß wir es mit relativ einheitlichem Material zu tun hatten.

Anschließend wurde der Rückstand 20 min lang mit konzentrierter Flußsäure behandelt. Hierbei gingen $33 \%$ der angesetzten Probe in Lösung. Es handelt sich dabei offenkundig um die Außenschicht der Pyroxenkörner, während die verbleibenden $67 \%$ das Innere darstellen. Die Ergebnisse der Edelgasanalysen vor und nach dem Einwirken der Flußsäure sind in Tab. 3 aufgeführt. Wie ersichtlich, hatten die Außenschichten etwa doppelt soviel Helium und mehr als viermal soviel Neon als das Innere der Körner. Wir können daher annehmen, daß die leichten Uredelgase ursprünglich allein in den äußersten Schichten saßen. Durch Diffusion ist dann Edelgas sowohl nach außen verlorengegangen als auch ins Korninnere gewandert, Helium natürlich stärker als Neon. Auch die ${ }^{4} \mathrm{He} /{ }^{3} \mathrm{He}-$ bzw. ${ }^{20} \mathrm{Ne} /{ }^{22} \mathrm{Ne}-V e r h a ̈ l t-$ nisse sind jeweils in der Richtung einer Diffusion von außen nach innen verschoben. In Tab. 3 sind nur die unkorrigierten Werte angegeben. Die tatsächliche Verschiebung der Isotopenverhältnisse ist noch um einiges höher.

Um bei der Metallphase analog nur die obersten Schichten zu lösen, haben wir Lösungsversuche mit Wasser angestellt. Hierbei stellten wir fest, daß das Nickeleisen bei kräftigem Rühren mittels eines Magnetrührers im Vakuum sowohl in mit einigen Trop- fen $\mathrm{H}_{2} \mathrm{SO}_{4}\left(p_{\mathrm{H}} 4\right)$ angesäuertem Wasser als auch in reinem Wasser in Spuren gelöst wird. Hierbei wird Wasserstoff entwickelt. Ohne Rühren geht hingegen kein Eisen in Lösung, auch wird kaum Uredelgas in Freiheit gesetzt.

Die in Lösung gegangene Eisenmenge chemisch zu bestimmen, erwies sich als schwierig, da das gelöste Eisen spätestens beim Abzentrifugieren des Meteoritpulvers teilweise wieder ausfällt. Wir haben sie daher aus der gebildeten Wasserstoffmenge abgeschätzt. Bezogen auf eine Einwaage von $1 \mathrm{~g}$ gingen in beiden Fällen (Schwefelsäure mit $p_{\mathrm{H}} 4 \mathrm{bzw}$. reines Wasser $p_{\mathrm{H}} 7$ ) jeweils etwa $10 \mathrm{mg}$ Eisen in Lösung. Hierbei wurde stets 12 Stunden lang unter ständigem, kräftigem Rühren gelöst. Die in Freiheit gesetzten Mengen von Urhelium betrugen 11800 $10^{-8} \mathrm{~cm}^{3}$ pro Gramm Einwaage bzw. $8730 \cdot 10^{-8}$ $\mathrm{cm}^{3}$ pro Gramm Einwaage. Im ersten Fall lieferte die gleiche Probe bei weiterem 12-stündigem Lösen mit neuer Lösung ( $p_{\mathrm{H}} 4$ ) nur noch $2940 \cdot 10^{-8} \mathrm{~cm}^{3}$ Helium pro Gramm Einwaage. Eine darauf anschließende Kupferchlorid-Extraktion brachte nunmehr $1100 \cdot 10^{-8} \mathrm{~cm}^{3}$ Helium pro Gramm Einwaage. Hiermit war eindeutig bewiesen, daß das durch die Wassereinwirkung in Freiheit gesetzte Uredelgas zum großen Teil den äußeren Schichten der Nickeleisenkörner entstammte. Bezogen auf $1 \mathrm{~g}$ gelöstes Eisen lieferten diese Extraktionen einen Wert von $10^{-2}$ $\mathrm{cm}^{3}$ Helium. Dies ist die höchste Anreicherung, die wir für den Meteoriten $\mathrm{P}$ a n tar gemessen hatten. Das gegenüber den Eisen-III-chlorid- bzw. KupferII-chlorid-Fraktionen in $\mathrm{Pantar} 3$ und $\mathrm{Pan}$ ta $\mathrm{r} 5$ auf durchschnittlich etwa 500 erniedrigte Verhältnis $\Sigma \mathrm{He} / \Sigma \mathrm{Ne}$ deutet jedoch darauf hin, daß noch zusätzlich Uredelgas aus anderen Meteoritbestandteilen in Freiheit gesetzt wurde. Bei dieser letzteren Komponente handelt es sich vermutlich um Edelgas, das in Spalten und Klüften von Silikatkörnern saß, wohin es durch Diffusion gelangte. Dies wird vor allem durch einen deutlichen Anteil

\begin{tabular}{|c|c|c|c|c|c|c|c|c|c|}
\hline & ${ }^{4} \mathrm{He}$ & \multicolumn{4}{|c|}{ in $10^{-8} \mathrm{~cm}^{3} / \mathrm{g}$} & \multicolumn{2}{|c|}{$\begin{array}{l}\text { Abreicherung } \\
\text { bzw. Anreicherung } \\
{ }^{4} \mathrm{He} \quad{ }^{20} \mathrm{Ne}\end{array}$} & $\frac{{ }^{4} \mathrm{He}}{{ }^{3} \mathrm{He}}$ & $\frac{{ }^{20} \mathrm{Ne}}{{ }^{22} \mathrm{Ne}}$ \\
\hline Ausgangsprobe & 27300 & 12,4 & 91,0 & 1,12 & 9,14 & 1,0 & 1,0 & 2200 & 9,96 \\
\hline Rückst. nach $\mathrm{HF}=$ Innen & 21100 & 10,2 & 42,3 & 1,01 & 4,00 & 0,77 & 0,46 & 2070 & 10,6 \\
\hline Rückst. $\times 0,67$ & 14100 & 6,8 & 28,4 & 0,67 & 2,68 & - & - & - & - \\
\hline Differenz zur Ausgangsprobe & 13200 & 5,6 & 62,6 & 0,45 & 6,46 & - & - & - & - \\
\hline Differenz: $0,33=$ Außenschicht & 40000 & 17,0 & 190 & 1,36 & 19,6 & 1,46 & 2,09 & 2360 & 9,68 \\
\hline
\end{tabular}

Tab. 3. Edelgasdaten von Pyroxen aus $\mathrm{P}$ a n t a r $3[<60 \mu]$. Vor und nach der Behandlung mit Flußsäure. 
an Spallations- ${ }^{21} \mathrm{Ne}$ angedeutet. Die gemessenen Isotopenverhältnisse dieser Extraktion mit Wasser waren mit ${ }^{4} \mathrm{He} /{ }^{3} \mathrm{He}=2300$ und ${ }^{20} \mathrm{Ne} /{ }^{22} \mathrm{Ne}=13,9$ recht ähnlich der in den Eisen-III-chlorid- und Kupfer-IIchlorid-Fraktionen gemessenen Verhältnisse.

\section{Zusammenfassung}

Unter Anwendung spezifischer Lösungsmittel zur Mineraltrennung konnte durch Edelgasmessungen an uredelgashaltigen Steinmeteoriten folgendes gezeigt werden:

1. Die leichten Uredelgase sind in allen Hauptmineralien der Meteorite enthalten. Dies gilt sicher für die Bronzit-Chondrite $\mathrm{Breitscheid}$ und $\mathrm{P}$ a n t a r und wahrscheinlich auch für den kohligen Chondrit Murray und den Achondriten Kap o eta.

2. Die leichten Uredelgase sind an den äußeren Schichten der einzelnen Mineralkörner hoch angereichert. Die Konzentrationen im Inneren der Körner sind gering und entsprechen wahrscheinlich Edelgasmengen, die von den Oberflächenschichten nach dem Inneren diffundiert sind.

3. Sowohl das ${ }^{4} \mathrm{He} /{ }^{3} \mathrm{He}$ - und ${ }^{20} \mathrm{Ne} /{ }^{22} \mathrm{Ne}-V e r h a ̈ l t-$ nis als auch das ${ }^{4} \mathrm{He} /{ }^{20} \mathrm{Ne}-V e r h a ̈ l t n i s$, wie es heute in den uredelgashaltigen Meteoriten gefunden wird, ist nicht nur von Meteorit zu Meteorit verschieden, sondern schwankt auch in den verschiedenen Mineralien innerhalb eines Meteoriten.
4. Das ${ }^{4} \mathrm{He} /{ }^{3} \mathrm{He}$-, das ${ }^{4} \mathrm{He} /{ }^{20} \mathrm{Ne}$ - und vor allem

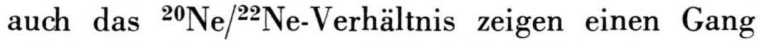
mit den Diffusionseigenschaften der Edelgase in den einzelnen Mineralien. Stets ist das leichtere Isotop in den Mineralien, die allgemein größere Uredelgasverluste zeigen, am meisten angereichert.

5. Aus den die Isotopenverschiebungen bedingenden Diffusionsverlusten ergibt sich für $\mathrm{Pantar}$ ein ursprünglicher Gehalt an Urhelium, der um etwa ein bis zwei Größenordnungen höher ist als der gegenwärtige.

6. Als beste Werte für die wahren primordialen Isotopen- und Elementverhältnisse gelten 13,8 bis 14,0 für das ${ }^{20} \mathrm{Ne} /{ }^{22} \mathrm{Ne}$-Verhältnis, 2200 für das ${ }^{4} \mathrm{He} /{ }^{3} \mathrm{He}-$ Verhältnis und 800 für das ${ }^{4} \mathrm{He} /{ }^{20} \mathrm{Ne}-\mathrm{Ver}$ -

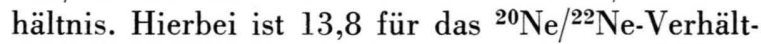
nis als untere Grenze anzusehen; der tatsächliche Wert liegt vermutlich bei 14,0. Die bisher angegebenen Werte für dieses Verhältnis, die aus Edelgasmessungen an den gesamten Meteoriten gewonnen wurden, sind durch Diffusionsverluste mehr oder minder verfälscht.

Die Frage des Ursprungs der leichten Uredelgase wird in einer folgenden Arbeit gesondert behandelt.

Für ihre Mithilfe bei der Ausführung der experimentellen Arbeiten danken wir Frl. H. Harasin, M. Monerjan und I. Schüler, und den Herren B. Spettel und B. Wellner. Die Arbeit wurde unterstützt durch Leihgaben der Deutschen Forschungsgemeinschaft und durch das Bundesministerium für wissenschaftliche Forschung. 